\title{
Article \\ A Possible Outbreak by Serratia marcescens: Genetic Relatedness between Clinical and Environmental Strains
}

\author{
Giuseppina Caggiano ${ }^{1, *}$ (D) Francesco Triggiano ${ }^{1}$, Giusy Diella ${ }^{1}$, Francesca Apollonio ${ }^{1}$, Marco Lopuzzo ${ }^{1}$,

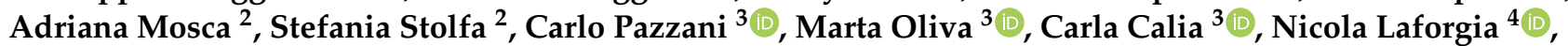 \\ Lidia Dalfino ${ }^{5}$, Giovanna Barbuti ${ }^{1}$, Pasquale Stefanizzi ${ }^{1}$, Anna Maria Minicucci ${ }^{6}$, Osvalda De Giglio ${ }^{1}$ (D) \\ and Maria Teresa Montagna ${ }^{1}[$
}

check for updates

Citation: Caggiano, G.; Triggiano, F.; Diella, G.; Apollonio, F.; Lopuzzo, M.; Mosca, A.; Stolfa, S.; Pazzani, C.; Oliva, M.; Calia, C.; et al. A Possible Outbreak by Serratia marcescens: Genetic Relatedness between Clinical and Environmental Strains. Int. J. Environ. Res. Public Health 2021, 18, 9814. https://doi.org/10.3390/ ijerph18189814

Academic Editor: Paul B. Tchounwou

Received: 15 August 2021

Accepted: 16 September 2021

Published: 17 September 2021

Publisher's Note: MDPI stays neutral with regard to jurisdictional claims in published maps and institutional affiliations.

Copyright: (c) 2021 by the authors. Licensee MDPI, Basel, Switzerland. This article is an open access article distributed under the terms and conditions of the Creative Commons Attribution (CC BY) license (https:// creativecommons.org/licenses/by/ $4.0 /)$
1 Department of Biomedical Science and Human Oncology, University of Bari Aldo Moro, Piazza G. Cesare 11, 70124 Bari, Italy; francesco.triggiano@uniba.it (F.T.); giusy.diella@uniba.it (G.D.); francesca.apo@libero.it (F.A.); marcolopuzzo@gmail.com (M.L.); giovanna.barbuti@uniba.it (G.B.); pasquale.stefanizzi@uniba.it (P.S.); osvalda.degiglio@uniba.it (O.D.G.); mariateresa.montagna@uniba.it (M.T.M.)

2 Department of Interdisciplinary Medicine, Microbiology Section, University of Bari Aldo Moro, Piazza G. Cesare 11, 70124 Bari, Italy; adriana.mosca@uniba.it (A.M.); stolfastefania@gmail.com (S.S.)

3 Department of Biology, University of Bari Aldo Moro, Via Orabona 4, 70125 Bari, Italy; carlo.pazzani@uniba.it (C.P.); marta.oliva@uniba.it (M.O.); carla.calia@uniba.it (C.C.)

4 Neonatology and Intensive Care Neonatal Unit Section, Department of Interdisciplinary Medicine, University of Bari Aldo Moro, Piazza G. Cesare 11, 70124 Bari, Italy; nicola.laforgia@uniba.it

5 Department of General Surgery, Gynecology and Anaesthesia-Anaesthesia and Intensive Care Unit II, University Hospital Policlinico of Bari, 70124 Bari, Italy; lidia.dalfino@yahoo.com

6 Health Management, University Hospital Policlinico of Bari, 70124 Bari, Italy; annamariaminicucci@gmail.com

* Correspondence: giuseppina.caggiano@uniba.it; Tel.: +39-(0)-80-5478-475

Abstract: Serratia marcescens (SM) is a Gram-negative bacterium that is frequently found in the environment. Since 1913, when its pathogenicity was first demonstrated, the number of infections caused by SM has increased. There is ample evidence that SM causes nosocomial infections in immunocompromised or critically ill patients admitted to the intensive care units (ICUs), but also in newborns admitted to neonatal ICUs (NICUs). In this study, we evaluated the possible genetic correlation by PFGE between clinical and environmental SM strains from NICU and ICU and compared the genetic profile of clinical strains with strains isolated from patients admitted to other wards of the same hospital. We found distinct clonally related groups of SM strains circulating among different wards of a large university hospital. In particular, the clonal relationship between clinical and environmental strains in NICU and ICU 1 was highlighted. The identification of clonal relationships between clinical and environmental strains in the wards allowed identification of the epidemic and rapid implementation of adequate measures to stop the spread of SM.

Keywords: Serratia marcescens; outbreak; intensive care unit; neonatal intensive care unit; genetic relatedness; pulsed-field gel electrophoresis; environmental sampling; strains

\section{Introduction}

Serratia marcescens (SM) is a Gram-negative bacterium belonging to the family Enterobacterales that is commonly found in water, soil, animals, and plants [1,2]. Although SM was previously considered a saprophytic microorganism, its pathogenicity was demonstrated in 1913 [1,3] but, unfortunately, its role in the etiology of human infectious diseases was underestimated for many years [3]. The first outbreak of nosocomial infection caused by SM was confirmed in 1951, which then aroused the interest of many researchers [3]. Later, reports of infections caused by SM increased. Some authors [1,3-5] have shown that SM causes nosocomial infections in immunocompromised or critically ill patients admitted to intensive care units (ICUs). Other authors have reported that SM colonizes the 
respiratory and urinary tracts, especially in the presence of devices such as catheters and endotracheal tubes in adult patients [6]. Newborns, especially those admitted to neonatal ICUs (NICUs), also represent an important reservoir of SM. Once infected, many infants have persistent intestinal colonization that can last for several years, despite antibiotic treatment $[1,7,8]$.

Because of its strong ability to adhere to invasive hospital equipment, SM can form biofilms and render conventional therapy ineffective [9], as a result of its intrinsic resistance to several classes of antibiotics (e.g., $\beta$-lactams and tetracyclines).

Data from the European Center for Disease Prevention and Control (ECDC) show that in 2017 Serratia spp. were ranked sixth among the ten most frequently isolated microorganisms in ICU-acquired pneumonia episodes (5.3\%) and ninth in bloodstream and urinary tract infections (3.4\%) in patients admitted to European ICUs [10].

The aims of this study were: (i) to evaluate the possible genetic correlation between clinical and environmental SM strains from the same hospital wards, and (ii) to compare the genetic profile of clinical strains with strains isolated from patients recovered in other wards of the same hospital.

\section{Materials and Methods}

From January to December 2020, 99 cases of SM infection occurred in a large Italian university hospital with 1400 beds in 33 separate buildings. The observational study involved patients admitted to internal medicine, endocrinology, infectious diseases, hematology, nephrology, general surgery, plastic surgery, cardiac surgery, and vascular surgery wards, NICU, ICU 1, and ICU 2; all located in different buildings. SM was isolated from 114 clinical samples, in particular: 46 (40.4\%) from respiratory tract, $26(22.8 \%)$ from urine, 15 (13.2\%) from blood, 9 (7.9\%) from effusion exudates, 6 (5.3\%) from throat swabs, $4(3.5 \%)$ from gastric aspirates, $3(2.6 \%)$ from intravascular catheters, $2(1.7 \%)$ from ocular conjunctiva, $2(1.7 \%)$ from prostatic secretions, and $1(0.9 \%)$ from cerebrospinal fluid. In most patients, SM was found in only one site but in 10 patients, SM was found in different sites.

In all wards, SM infections occurred sporadically except for those in the NICU and ICU 1. According to hospital protocols, in these cases (i.e., more than two episodes of illness caused by the same microorganism within a month in the same ward), it is mandatory to carry out environmental sampling to evaluate the possible presence of the same microorganism in the environment, especially on surfaces or equipment. Consequently, an environmental investigation was carried out in the NICU and ICU 1 to identify the possible environmental reservoir of infection.

\subsection{Serratia marcescens in NICU and ICU}

In September 2020, a newborn admitted to the NICU developed bacterial sepsis. Blood culture and central venous catheter (CVC) both tested positive for SM. The baby was preterm (25 weeks' gestation) and, despite early antibiotic treatment, the newborn died 5 days after diagnosis of SM sepsis. During the same period in the same NICU, another preterm newborn developed SM sepsis that resolved after antibiotic therapy. At the same time, four patients developed SM infections in ICU 1. The microorganism was isolated from tracheal aspirates of three patients and from urine of the other patient.

The search for SM was performed by plating a loop of the biological sample on MacConckey Agar (Biolife Italiana srl, Milan, Italy) and incubating the plates at $37 \pm 1{ }^{\circ} \mathrm{C}$ for 18-24 h. Identification of suspicious colonies was performed by matrix-assisted laser desorption/ionization time-of-flight mass spectrometry (MALDI-TOF MS, Biomèrieux, Marcy l'Etoile, France).

\subsection{Environmental Sampling}

One hundred and fifteen environmental samples were collected; 75 from the NICU (walls, incubators, changing tables, therapeutic trolleys, medical tools prewash brushes, 
washbasins, and jet breakers) and 40 from ICU 1 (walls, beds, bedside tables, therapy trolleys, sinks, and book supports). The sampling was performed by sterile swabs inserted in a plastic tube (Easy Surface Checking-Neutralizing Rinse Solution; Liofilchem Srl, Roseto degli Abruzzi, Italy) containing $10 \mathrm{~mL}$ transport medium, according to recommendations of the European Standard-International Organization for Standardization [11].

The flat and wide surfaces (regular surfaces, e.g., beds, bedside shelves, and incubators), were sampled with a swab on a well-defined area $(10 \times 10 \mathrm{~cm})$, using a delimiter, while for small surfaces and curves (irregular surfaces, e.g., catheters, endotracheal tubes, and jet breakers) the available area was sampled. The swabs were placed in their protective cases, transported to the laboratory in refrigerated containers at $4{ }^{\circ} \mathrm{C}$, and immediately tested. All samples were vortexed for $20 \mathrm{~s}$ at room temperature, and 1-mL aliquots of liquid transport medium of each dilution were mixed separately with Plate Count Agar (Microbiol Snc, Cagliari, Italy) to evaluate total microbial count, incubated at $30 \pm 1{ }^{\circ} \mathrm{C}$ and checked daily for growth for $72 \pm 3 \mathrm{~h}$ [12]. Colony counts were enumerated as colony-forming units (cfu) per $\mathrm{cm}^{2}$ (regular surface) or cfu/swab (irregular surface). At the same time, the search for SM was performed by plating a loop of the transport medium on Wurtz Agar (Biolife Italiana srl, Milan, Italy) and incubating the plates at $37 \pm 1{ }^{\circ} \mathrm{C}$ for $24-48 \mathrm{~h}$. Identification of suspicious colonies was performed by MALDI-TOF MS.

\subsection{Antibiotic Susceptibility}

The VITEK ${ }^{\circledR} 2$ automated identification system AST-N397 card (Biomèrieux, Marcy l'Etoile, France) was used to determine the antibiotic susceptibility patterns for amikacin, cefepime, ceftazidime/avibactam, ceftolozane/tazobactam, ciprofloxacin, colistin, gentamicin, imipenem, meropenem, tobramycin, and trimethoprim/sulfamethoxazole. Results were interpreted according to identification criteria set for Serratia marcescens by the European Committee on Antimicrobial Susceptibility Testing-EUCAST. The clinical and environmental strains were all sensitive to the antimicrobial drug classes tested.

\subsection{Molecular Investigation}

Molecular investigations by pulsed-field gel electrophoresis (PFGE) were carried out on clinical and environmental strains isolated in the NICU and ICU 1, and on other clinical isolates from patients admitted to different wards of the same hospital and available in our stock library. Four SM strains were isolated in ICU 2 (two from tracheal aspirates, and one each from bronchoalveolar lavage (BAL) and bronchial excretion); three in cardiac surgery (from blood cultures); and one in internal medicine (from blood cultures). Finally, two SM strains were isolated from BAL of patients admitted to general surgery.

\subsection{PFGE}

Genomic restrictions were performed as previously reported [13] and according to the standard operating procedure for PulseNet PFGE of Escherichia coli O157:H7, E. coli non-O157 (STEC), Salmonella serotypes, Shigella sonnei, and Shigella flexneri [14]. Agaroseembedded DNA was digested with $40 \mathrm{U} X b a \mathrm{I}$ for $4 \mathrm{~h}$ at $37^{\circ} \mathrm{C}$. The fragments were separated in 1\% agarose gel (Pulsed Field Certified Agarose; BioRad, Milan, Italy) in Tris-borateEDTA (44.5 mM Tris-borate, $1 \mathrm{mM}$ EDTA; pH 8.0) at $14{ }^{\circ} \mathrm{C}$, using a CHEF-DRIII (Bio-Rad, Milan, Italy) apparatus. Electrophoresis conditions were as follows: initial switch time $7.3 \mathrm{~s}$, final switch time $24 \mathrm{~s}$, voltage $6 \mathrm{~V}$, included angle $120^{\circ}$, and run time $19 \mathrm{~h}$. XbaI-digested DNA fragments from the Salmonella Braenderup strain H9812 were used in each gel as universal size standards. The agarose gels were stained with ethidium bromide $(40 \mathrm{~g} / \mathrm{mL})$ and the DNA band images were acquired by the Gel Doc-It photo documentation system (UVP, Upland, CA, USA). 


\subsection{Genetic Relatedness}

Genetically related isolates (clones) were indistinguishable from each other by genetic tests such as PFGE or were so similar that they were presumed to be derived from a common parent [15].

PFGE profiles were analyzed using the fingerprinting software GelJ [16]. Phylogenetic trees were constructed using the Dice coefficient with clustering by the unweighted pair-group method with arithmetic mean (UPGMA) and $2 \%$ tolerance in band position differences. The normalization of every gel image was performed using the XbaI-digested DNA fragments from $S$. Braenderup strain H9812 as an external reference. For determination of genotype diversity, a cutoff value of $80 \%$ was used [17] to interpret minimum similarity, while the strains were considered to belong to the same clonal group when PFGE profiles had a value of similarity $\geq 95 \%$. The genetic correlation was first assessed on strains isolated from clinical and environmental samples in the NICU and ICU 1. These profiles were correlated with those of other clinical strains from patients in different wards of the same hospital.

The DNA restriction pattern that was designated the outbreak pattern was reported as type A; the isolates whose restriction patterns were indistinguishable from that pattern were reported as representing the outbreak strain. Patterns that were closely or possibly related to the outbreak pattern were considered as subtypes of $A$ and were designated type A1, type A2, etc. Patterns that differed substantially from the outbreak pattern and were classified as unrelated were designated type B, type C, etc. [15].

\section{Results}

From environmental investigations carried out in the NICU and ICU 1 , three swabs $(4 \%, 3 / 75)$ in the NICU were positive for SM and two $(5 \%, 2 / 40)$ in ICU 1 . In the NICU, one strain was isolated from a brush used before sterilization of surgical forceps and two from washbasins, while in ICU 1, the two strains were isolated from a book support and a faucet handle.

Overall, 22 SM strains were subjected to molecular investigations: 17 clinical strains (three from the NICU, four from ICU 1, and 10 from other wards) and five environmental strains (three from the NICU and two from ICU 1) (Table 1).

Table 1. Serratia marcescens isolated from clinical and environmental samples in different wards.

\begin{tabular}{lcc}
\hline SM Strain & Examined Sample & Ward \\
\hline cs-SM/04 & Blood culture & NICU \\
es-SM/05 & Washing brush & NICU \\
cs-SM/06 & CVC & NICU \\
cs-SM/13 & Tracheal aspirate & ICU 2 \\
cs-SM/10 & BAL & General surgery \\
cs-SM/14 & Tracheal aspirate & ICU 2 \\
es-SM/18 & Tracheal aspirate & ICU 1 \\
es-SM/19 & Faucet handle & ICU 1 \\
cs-SM/20 & Book support & ICU 1 \\
cs-SM/03 & Blood culture & Cardiac surgery \\
cs-SM/12 & Blood culture & NICU \\
cs-SM/15 & BAL & ICU 2 \\
es-SM/01 & ICU 2 \\
cs-SM/02 & Bronchial excreted & NICU \\
cs-SM/08 & Washbasin & NICU \\
cs-SM/09 & Washbasin & Cardiac surgery \\
cs-SM/21 & Blood culture & Internal medicine \\
cs-SM/11 & Blood culture & Cardiac surgery \\
cs-SM/16 & Blood culture & ICU 1 \\
cs-SM/22 & Tracheal aspirate & ICU 1 \\
\hline
\end{tabular}

BAL, bronchoalveolar lavage; cs-SM: clinical sample-Serratia marcescens; CVC, central venous catheter; es-SM: environmental sample-Serratia marcescens; /No.: identification number of the studied strains, according to our classification. 


\section{PFGE and Genetic Relatedness}

Comparing SM clinical and environmental strains, based on the lower limit of similarity of $80 \%$, 19 strains were grouped into three PFGE groups, designated A, B, and C, and in clonal subgroups A1, B1, B2, and C1 (Table 2).

Table 2. PFGE grouping and \% similarity of clinical and environmental strains of Serratia marcescens according to sample and ward.

\begin{tabular}{|c|c|c|c|c|c|}
\hline PFGE Group & PFGE Subgroup & SM Strain & Examined Sample & Ward & $\%$ Similarity \\
\hline $\mathrm{A}$ & A1 & cs-SM/04 & Blood culture & NICU & \multirow{4}{*}{100} \\
\hline $\mathrm{A}$ & A1 & es-SM/05 & Washing brush & NICU & \\
\hline $\mathrm{A}$ & A1 & cs-SM/06 & CVC & NICU & \\
\hline $\mathrm{A}$ & None & cs-SM/13 & Tracheal aspirate & ICU 2 & \\
\hline B & B1 & cs-SM/10 & BAL & General surgery & \multirow{3}{*}{95} \\
\hline B & B1 & cs-SM/17 & Tracheal aspirate & ICU 2 & \\
\hline B & B2 & cs-SM/14 & Tracheal aspirate & ICU 1 & \\
\hline B & B2 & es-SM/18 & Faucet handle & ICU 1 & \multirow[t]{2}{*}{$\geq 96$} \\
\hline B & B2 & es-SM/19 & Book support & ICU 1 & \\
\hline B & B2 & cs-SM/20 & Blood culture & Cardiac surgery & \multirow[t]{4}{*}{100} \\
\hline B & None & cs-SM/03 & Blood culture & NICU & \\
\hline B & None & cs-SM/12 & BAL & ICU 2 & \\
\hline B & None & cs-SM/15 & Bronchial excreted & ICU 2 & \\
\hline $\mathrm{C}$ & $\mathrm{C} 1$ & es-SM/01 & Washbasin & NICU & \multirow{7}{*}{97} \\
\hline $\mathrm{C}$ & $\mathrm{C} 1$ & es-SM/02 & Washbasin & NICU & \\
\hline $\mathrm{C}$ & None & cs-SM/07 & Blood culture & Cardiac surgery & \\
\hline $\mathrm{C}$ & None & cs-SM/08 & Blood culture & Internal medicine & \\
\hline $\mathrm{C}$ & None & cs-SM/09 & Blood culture & Cardiac surgery & \\
\hline $\mathrm{C}$ & None & cs-SM/21 & Tracheal aspirate & ICU 1 & \\
\hline Unique & None & cs-SM/11 & Tracheal aspirate & ICU 1 & \\
\hline Unique & None & cs-SM/16 & BAL & General surgery & \\
\hline Unique & None & cs-SM/22 & Urine culture & ICU 1 & \\
\hline
\end{tabular}

BAL, bronchoalveolar lavage; cs-SM: clinical sample-Serratia marcescens; CVC, central venous catheter; es-SM: environmental sample-Serratia marcescens; / No.: Identification No. of Serratia marcesens strains; PFGE, pulsed-field gel electrophoresis.

Group A consisted of four strains, of which three (subgroup A1) were indistinguishable from each other: two clinical strains from NICU patient (blood culture and CVC) and an environmental strain isolated from a medical tools prewash brush from the same ward (100\% similarity).

Group B consisted of nine strains: two in subgroup B1 and four in subgroup B2 (95\% and $\geq 96 \%$ similarity, respectively). Subgroup B1 consisted of strains reported as cs-SM/10 and cs-SM/17 isolated from recovered patients in two different wards (general surgery and ICU 2). Subgroup B2 consisted of three strains indistinguishable from each other (100\% similarity) and reported as cs-SM/14, es-SM/18, and es-SM/19. These strains came from ICU 1: two (es-SM/18 and es-SM/19) were detected from environmental samples (faucet handle and book support, respectively) and one (cs-SM/14) from tracheal aspirate. The fourth strain (cs-SM/20) was isolated from blood culture of a patient recovered in cardiac surgery.

Group C consisted of six strains. Two of environmental origin (es-SM/01 and es$\mathrm{SM} / 02$, from two different washbasins in the NICU) constituted subgroup C1 (97\% similarity). The other four strains, isolated from patients (blood culture and tracheal aspirate) admitted to different wards, did not belong to any PFGE subgroup.

The last three strains (cs-SM/11, cs-SM/16 and cs-SM/22; all clinical strains) were labeled as unique PFGE profiles since their similarity to PFGE was $\leq 79 \%$. In particular, cs$\mathrm{SM} / 11$ and cs-SM/22 were isolated from tracheal aspirate and urine of patients recovered in ICU 1, and cs-SM/16 was isolated from BAL of a patient in general surgery (Figure 1). 


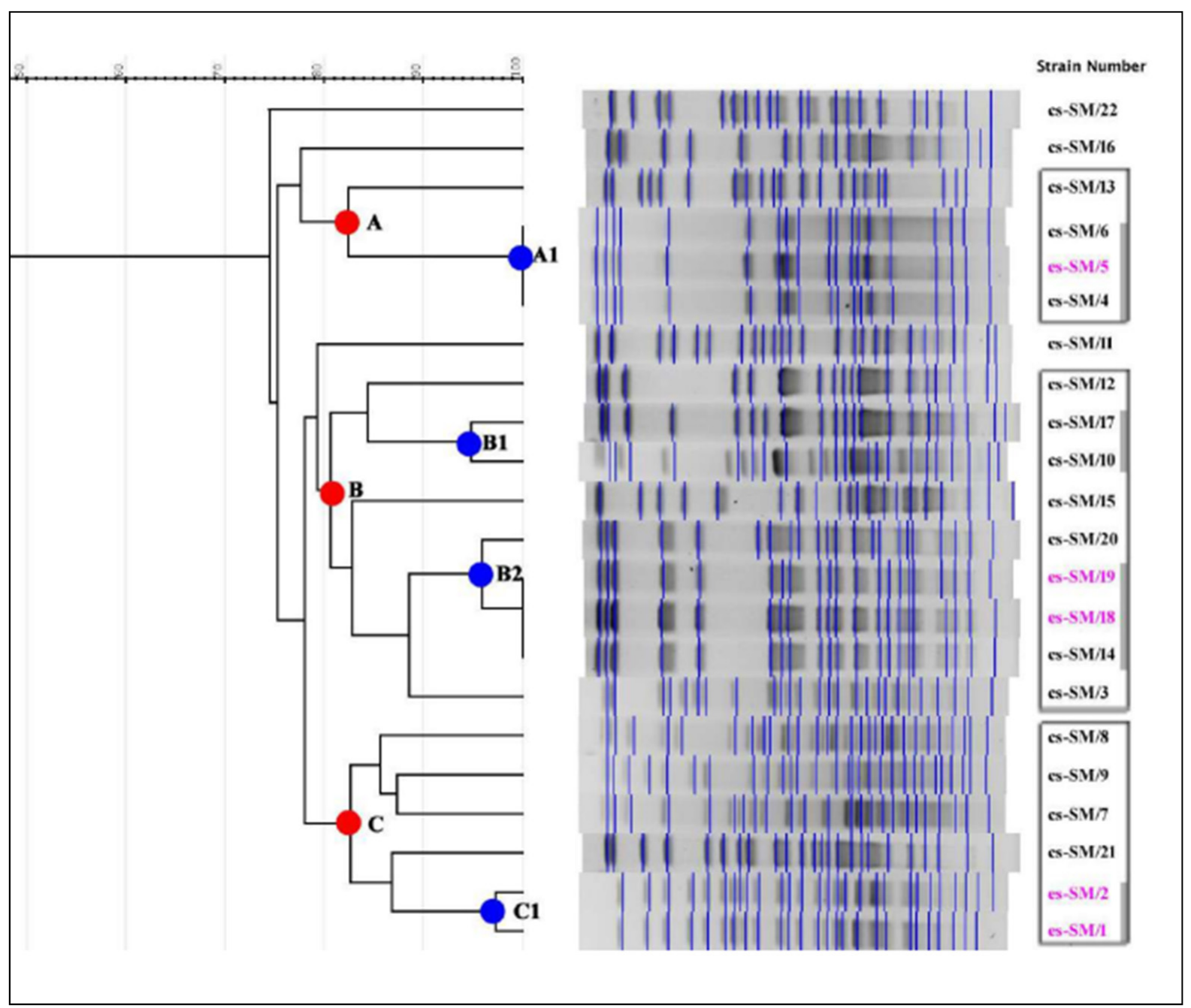

Figure 1. Pulsed-field gel electrophoresis generated dendrogram for 17 clinical and five environmental Serratia marcescens strains. Legend cs-SM (black color) = clinical strain-S. marcescens. es-SM (fuchsia color) = environmental strain-S. marcescens.

\section{Discussion}

Although SM is an environmental saprophytic microorganism, it can cause dangerous nosocomial diseases in immunocompromised patients, as well as in wound and soft tissue infections [18], open burn surgical wound infections [19], and invasive burn wound infections [20].

Our study highlights distinct clonally related groups (A-C) of SM circulating between different wards and in different areas of the same hospital. The identification of three indistinguishable strains (subgroup A1) in the NICU, isolated from the CVC and blood culture of the same patient and from a medical tools prewash brush, emphasizes the possible cross-contamination between personnel and the environment, and how the latter may represent a potential source of microbial spread and a threat to human health. However, both clinical and environmental strains unrelated to subgroup A1 were also isolated in the same ward. A similar finding was observed in ICU 1 where indistinguishable strains (subgroup B2) from tracheal aspirates, faucet handles, and book supports and clinical strains unrelated to subgroup B2 were detected.

The presence of strains related to subgroups B1 and B2 was also highlighted in other wards. Strains cs-SM/10 and cs-SM/17, clonally related to subgroup B1, were detected in ICU 2 and general surgery wards, respectively, and cs-SM/20, clonally related to the B2 subgroup, was detected in cardiac surgery. 
These data suggest the circulation in different wards of the same hospital of both clonally distinct and clonally related strains. The latter hypothesis could be consistent with the presence of environmental niches that act as reservoirs and sources of bacterial transmission. It would be interesting to investigate if this strain clonal relatedness might somehow be related to environmental and/or genetic features yet to be identified

The mode of transmission of SM from surfaces or patients is not easy to demonstrate. Cross-infection of SM, mainly from contaminated hands of hospital staff, has been highlighted for many years, as have environmental sources as risk factors for SM outbreaks [21-23]. The invasive disease may have been facilitated by the presence of CVC and endotracheal tubes that favor bacterial proliferation. Other authors [24,25] have shown that $\mathrm{SM}$ is ubiquitous and resistant to disinfection and can survive on inanimate surfaces, such as medical devices, and even in antiseptic solutions, for long periods.

Our study highlighted the isolation of SM from medical prewash brush. Although there is no certainty that they are a source of infection, careful epidemiological investigation and environmental surveillance can prevent the transmission of SM to other patients and prevent the onset of dangerous outbreaks. In our case, the brush was carefully washed under running water and disinfected after use. This procedure was probably not sufficient to eradicate any contaminants, or the disinfectant used was not suitable for eliminating $\mathrm{SM}$. We are carrying out some tests to resolve this doubt, in accordance with previous studies [26]. Furthermore, the brush was made from plastic material and previous studies have shown that plastic, being a porous material, can retain both bacteria and SARS$\mathrm{CoV}-2$, and their persistence on this material lasts for several months [27,28]. Therefore, appropriate disinfection protocols are required.

We believe that the conscious choice of a disinfectant and its use in compliance with the procedures indicated by the manufacturer represent a further intervention to minimize infections related to health care.

Our study has some limitations such as: not having identified the source of infection and the use of PFGE which, despite its high discriminatory power, has long analysis times, often poor inter-gel reproducibility, and requires specific equipment for electrophoresis and computerized image analysis. In our opinion, to control the spread of SM in the hospital setting and to safeguard the health of patients, especially if immunocompromised, targeted preventive measures, such as patient isolation, dedicated hospital staff, and proper hand hygiene are mandatory. Furthermore, environmental microbiological surveillance should always be scheduled, especially during an outbreak, by intensifying the cleaning and sanitizing protocols regarding surfaces and medical equipment $[29,30]$. In this regard, the conscious choice of a disinfectant and its use in compliance with the procedures indicated by the manufacturer represent a further intervention to minimize infections related to health care [26].

\section{Conclusions}

SM infections currently represent a serious challenge in hospital settings, especially in high-risk wards, such as NICUs and adult ICUs. The identification of clonal relationships by PFGE analysis between clinical and environmental strains allows the rapid implementation of measures to stop the spread of SM. Surveillance programs and proper disinfection procedures are indispensable. Among the preventive interventions, hand hygiene appears to be of particular importance as contaminated hands are a relevant route of transmission. Additionally, the molecular characterization of the strain is essential to detect outbreaks and identify possible sources of infection. The training of health workers on the issues of hand hygiene, use of personal protective equipment, and environmental hygiene are key to reducing the incidence of hospital infections.

Author Contributions: Conceptualization, M.T.M., G.C. and O.D.G.; data curation, G.D., F.A.; software, formal analysis P.S., G.B.; project administration, M.T.M., G.C.; environmental investigation M.L., F.T., molecular investigation C.P., M.O. and C.C.; microbiological clinical investigation A.M., S.S., G.D. and F.A.; writing—original draft preparation M.T.M., G.C. and F.T.; clinical investigation, 
N.L., L.D.; visualization A.M.M. All authors have read and agreed to the published version of the manuscript.

Funding: This research received no external funding.

Institutional Review Board Statement: Not applicable.

Informed Consent Statement: Not applicable.

Data Availability Statement: Not applicable.

Conflicts of Interest: The authors declare no conflict of interest.

\section{References}

1. Cristina, M.L.; Sartini, M.; Spagnolo, A.M. Serratia marcescens infections in neonatal intensive care units (NICUs). Int. J. Environ. Res. Public Health 2019, 16, 610. [CrossRef] [PubMed]

2. Scrascia, M.; Pazzani, C.; Valentini, F.; Oliva, M.; Russo, V.; D'Addabbo, P.; Porcelli, F. Identification of pigmented Serratia marcescens symbiotically associated with Rhynchophorus ferrugineus Olivier (Coleoptera: Curculionidae). MicrobiologyOpen 2016, 5, 883-890. [CrossRef] [PubMed]

3. Al Jarousha, A.M.; El Qouqa, A.; El Jadba, A.H.; Al Afifi, A.S. An outbreak of Serratia marcescens septicaemia in neonatal intensive care unit in Gaza City, Palestine. J. Hosp. Infect. 2008, 70, 119-126. [CrossRef] [PubMed]

4. Dessì, A.; Puddu, M.; Testa, M.; Marcialis, M.A.; Pintus, M.C.; Fanos, V. Serratia marcescens infections and outbreaks in neonatal intensive care units. J. Chemother. 2009, 21, 493-499. [CrossRef]

5. Uduman, S.A.; Farrukh, A.S.; Nath, K.N.R.; Zuhair, M.Y.; Ifrah, A.; Khawla, A.D.; Sunita, P. An outbreak of Serratia marcescens infection in a special-care baby unit of a community hospital in United Arab Emirates: The importance of the air conditioner duct as a nosocomial reservoir. J. Hosp. Infect. 2002, 52, 175-180. [CrossRef]

6. Eisenstein, B.I.; Zaleznik, D.F. Enterobacteriaceae. In Principles and Practice of Infectious Diseases, 5th ed.; Mandell, G.L., Bennett, J.E., Dolin, R., Eds.; Churchill Livingstone: Philadelphia, PA, USA, 2000; pp. 2294-2310.

7. Montagnani, C.; Cocchi, P.; Lega, L.; Campana, S.; Biermann, K.P.; Braggion, C.; Pecile, P.; Chiappini, E.; de Martino, M.; Galli, L. Serratia marcescens outbreak in a neonatal intensive care unit: Crucial role of implementing hand hygiene among external consultants. BMC Infect. Dis. 2015, 15, 11. [CrossRef]

8. David, M.D.; Weller, T.M.A.; Lambert, P.; Fraise, A.P. An outbreak of Serratia marcescens on the neonatal unit: A tale of two clones. J. Hosp. Infect. 2006, 63, 27-33. [CrossRef]

9. Rodrigues, A.P.; Holanda, A.R.; Lustosa, G.P.; Nóbrega, S.M.; Santana, W.J.; Souza, L.B.; Coutinho, H.D. Virulence factors and resistance mechanisms of Serratia marcescens. A short review. Acta Microbiol. Immunol. Hung. 2006, 53, 89-93. [CrossRef]

10. European Centre for Disease Prevention and Control. Healthcare-associated infections acquired in intensive care units. In ECDC. Annual Epidemiological Report for 2017; ECDC: Stockholm, Sweden, 2019. Available online: https:/ /www.ecdc.europa.eu/sites / default/files/documents / AER_for_2017-HAI.pdf (accessed on 14 May 2021).

11. ISO 14698-1: 2003 (en). Cleanrooms and Associated Controlled Environments Biocontamination Control-Part 1: General Principles and Methods; International Organization for Standardization: Geneva, Switzerland, 2003.

12. UNI EN ISO 4833-1:2013 (en). Horizontal Method for the Enumeration of Microorganisms. Part 1: Colony Count at 30 Degrees C by the Pour Plate Technique; International Organization for Standardization: Milan, Italy, 2013.

13. Scrascia, M.; D’Addabbo, P.; Roberto, R.; Porcelli, F.; Oliva, M.; Calia, C.; Dionisi, A.M.; Pazzani, C. Characterization of CRISPR-Cas Systems in Serratia marcescens Isolated from Rhynchophorus ferrugineus (Olivier, 1790) (Coleoptera: Curculionidae). Microorganisms 2019, 7, 368. [CrossRef]

14. Ribot, E.M.; Fair, M.A.; Gautom, R.; Cameron, D.N.; Hunter, S.B.; Swaminathan, B.; Barrett, T.J. Standardization of pulsed-field gel electrophoresis protocols for the subtyping of Escherichia coli O157:H7, Salmonella, and Shigella for PulseNet. Foodbourne Pathog. Dis. 2006, 3, 59-67. [CrossRef]

15. Tenover, F.C.; Arbeit, R.D.; Goering, R.V.; Mickelsen, P.A.; Murray, B.E.; Persing, D.H.; Swaminathan, B.J. Interpreting chromosomal DNA restriction patterns produced by pulsed-field gel electrophoresis: Criteria for bacterial strain typing. Clin. Microbiol. 1995, 33, 2233-2239. [CrossRef]

16. Heras, J.; Domínguez, C.; Mata, E.; Pascual, V.; Lozano, C.; Torres, C.; Zarazaga, M. GelJ-A tool for analyzing DNA fingerprint gel images. BMC Bioinform. 2015, 16, 270. [CrossRef] [PubMed]

17. van Belkum, A.; Tassios, P.T.; Dijkshoorn, L.; Haeggman, S.; Cookson, B.; Fry, N.K.; Fussing, V.; Green, J.; Feil, E.; Gerner-Smidt, P.; et al. Guidelines for the validation and application of typing methods for use in bacterial epidemiology. Clin. Microbiol. Infect. 2007, 13, 1-46. [CrossRef]

18. Us, E.; Kutlu, H.H.; Tekeli, A.; Ocal, D.; Cirpan, S.; Memikoglu, K.O. Wound and soft tissue infections of Serratia marcescens in patients receiving wound care: A health care-associated outbreak. Am. J. Infect. Control. 2017, 45, 443-447. [CrossRef]

19. Posluszny, J.A., Jr.; Conrad, P.; Halerz, M.; Shankar, R.; Gamelli, R.L. Surgical burn wound infections and their clinical implications. J. Burn Care Res. 2011, 32, 324-333. [CrossRef] 
20. Church, D.; Elsayed, S.; Reid, O.; Winston, B.; Lindsay, R. Burn wound infections. Clin. Microbiol. Rev. 2006, $19,403-434$. [CrossRef]

21. Jang, T.N.; Fung, C.P.; Yang, T.L.; Shen, S.H.; Huang, C.S.; Lee, S.H. Use of pulsed-field gel electrophoresis to investigate an outbreak of Serratia marcescens infection in a neonatal intensive care unit. J. Hosp. Infect. 2001, 48, 13-19. [CrossRef] [PubMed]

22. Alfizah, H.; Nordiah, A.J.; Rozaidi, W.S. Using pulsed-field gel electrophoresis in the molecular investigation of an outbreak of Serratia marcescens infection in an intensive care unit. Singap. Med. J. 2004, 45, 214-218.

23. Shi, Z.Y.; Liu, P.Y.; Lau, Y.J.; Lin, Y.H.; Hu, B.S. Use of pulsed-field gel electrophoresis to investigate an outbreak of Serratia marcescens. J. Clin. Microbiol. 1997, 35, 325-327. [CrossRef] [PubMed]

24. Moles, L.; Gómez, M.; Moroder, E.; Jiménez, E.; Escuder, D.; Bustos, G.; Melgar, A.; Villa, J.; Del Campo, R.; Chaves, F.; et al. Serratia marcescens colonization in preterm neonates during their neonatal intensive care unit stay. Antimicrob. Resist. Infect. Control 2019, 8, 135. [CrossRef] [PubMed]

25. Faro, J.; Katz, A.; Berens, P.; Ross, P.J. Premature termination of nursing secondary to Serratia marcescens breast pump contamination. Obstet. Gynecol. 2011, 117, 485-486. [CrossRef] [PubMed]

26. Montagna, M.T.; Triggiano, F.; Barbuti, G.; Bartolomeo, N.; De Giglio, O.; Diella, G.; Lopuzzo, M.; Rutigliano, S.; Serio, G.; Caggiano, G. Study on the in vitro activity of five disinfectants against nosocomial bacteria. Int. J. Environ. Res. Public Health 2019, 16, 1895. [CrossRef] [PubMed]

27. Gholipour, S.; Nikaeen, M.; Mohammadi Manesh, R.; Aboutalebian, S.; Shamsizadeh, Z.; Nasri, E.; Mirhendi, H. Severe Acute Respiratory Syndrome Coronavirus 2 (SARS-CoV-2) Contamination of High-touch Surfaces in Field Settings. Biomed. Environ. Sci. 2020, 33, 925-929. [PubMed]

28. Heller, L.C.; Edelblute, C.M. Long-term metabolic persistence of gram-positive bacteria on health care-relevant plastic. Am. J. Infect. Control. 2018, 46, 50-53. [CrossRef]

29. Redondo-Bravo, L.; Gutiérrez-González, E.; San Juan-Sanz, I.; Fernández-Jiménez, I.; Ruiz-Carrascoso, G.; Gallego-Lombardo, S.; Sánchez-García, L.; Elorza-Fernández, D.; Pellicer-Martínez, A.; Omeñaca, F.; et al. Serratia marcescens outbreak in a neonatology unit of a Spanish tertiary hospital: Risk factors and control measures. Am. J. Infect. Control 2019, 47, 271-279. [CrossRef] [PubMed]

30. Yeo, K.T.; Octavia, S.; Lim, K.; Lin, C.; Lin, R.; Thoon, K.C.; Tee, N.W.S.; Yung, C.F. Serratia marcescens in the neonatal intensive care unit: A cluster investigation using molecular methods. J. Infect. Public Health 2020, 13, 1006-1011. [CrossRef] 